\title{
$r$-Modified Crank-Nicholson difference scheme for fractional parabolic PDE
}

\author{
Allaberen Ashyralyev ${ }^{1,2}$ and Zafer Cakir ${ }^{3 *}$
}

${ }^{\text {*Correspondence: }}$

zafer@gumushane.edu.tr

${ }^{3}$ Department of Mathematical Engineering, Gumushane University,

Gumushane, Turkey

Full list of author information is

available at the end of the article

\begin{abstract}
The second order of accuracy stable difference scheme for the numerical solution of the mixed problem for the fractional parabolic equation are presented using by $r$-modified Crank-Nicholson difference scheme. Stability estimate for the solution of this difference scheme is obtained. A procedure of modified Gauss elimination method is used for solving this difference scheme in the case of one-dimensional fractional parabolic partial differential equations. Numerical results for this scheme and the Crank-Nicholson scheme are compared in test examples.
\end{abstract}

\section{Introduction}

At present, there is a huge number of theoretical and applied works devoted to the study of fractional differential equations. Solutions of various problems for fractional differential equations can be found, for example, in the monographs of Podlubny [1], Kilbas, Srivastava, and Trujillo [2], Diethelm [3], and in [4-11]. These problems were studied in various directions: qualitative properties of solutions, spectral problems, various statements of boundary value problems, and numerical investigations.

Many problems in fluid flow, dynamical and diffusion processes, control theory, mechanics, and other areas of physics can be reduced fractional partial differential equations.

In [12] the simple connection of fractional derivatives with fractional powers of first order differential operator was presented. This approach is important to obtain the formula for the fractional difference derivative. Presently, many mathematicians apply this approach and operator tools to investigate various problems for fractional partial differential equations which appear in applied problems (see, e.g., [13-20] and the references therein).

In previous paper [17] authors investigated stability estimates for Crank-Nicholson schemes for the Dirichlet problem for the fractional parabolic equation

$$
\left\{\begin{array}{l}
\frac{\partial u(t, x)}{\partial t}+D_{t}^{1 / 2} u(t, x)-\sum_{p=1}^{m}\left(a_{p}(x) u_{x_{p}}\right)_{x_{p}}+\sigma u(t, x)=f(t, x) \\
\quad x=\left(x_{1}, \ldots, x_{m}\right) \in \Omega, 0<t<T \\
u(t, x)=0, \quad x \in S \\
u(0, x)=0, \quad x \in \bar{\Omega} .
\end{array}\right.
$$

Here $D_{t}^{1 / 2}=D_{0+}^{1 / 2}$ is the standard Riemann-Liouville's derivative of order $1 / 2$ and $\Omega$ is the open cube in the $m$-dimensional Euclidean space

$$
\mathbb{R}^{m}:\left\{x \in \Omega: x=\left(x_{1}, \ldots, x_{m}\right) ; 0<x_{p}<1,1 \leq p \leq m\right\}
$$

○2014 Ashyralyev and Cakir; licensee Springer. This is an Open Access article distributed under the terms of the Creative Commons Attribution License (http://creativecommons.org/licenses/by/2.0), which permits unrestricted use, distribution, and reproduction in any medium, provided the original work is properly cited. 
with boundary $S, \bar{\Omega}=\Omega \cup S, a_{p}(x)(x \in \Omega)$ and $f(t, x)(t \in(0, T), x \in \Omega)$ are given smooth functions and $a_{p}(x) \geq a>0, \sigma>0$.

In [18] the authors investigated stability estimates for Crank-Nicholson schemes for the Neumann problem for the fractional parabolic equation

$$
\left\{\begin{array}{l}
\frac{\partial u(t, x)}{\partial t}+D_{t}^{1 / 2} u(t, x)-\sum_{p=1}^{m}\left(a_{p}(x) u_{x_{p}}\right)_{x_{p}}+\sigma u(t, x)=f(t, x) \\
\quad x=\left(x_{1}, \ldots, x_{m}\right) \in \Omega, 0<t<T \\
u(t, x)=0, \quad x \in S \\
\frac{\partial u(t, x)}{\partial \bar{n}}=0, \quad x \in \bar{\Omega} .
\end{array}\right.
$$

The role played by stability inequalities (well posedness) in the study of boundary-value problems for parabolic partial differential equations is well known (see, e.g., [21-26]).

In the present paper, we consider an $r$-modified Crank-Nicholson difference scheme of the above mentioned two problems (1.1), (1.2). This $r$-modified scheme is of the second order of accuracy in $t$ and in space variables difference schemes for the approximate solution of problems. The stability estimate for the solution of this difference scheme is established. We use a procedure of a modified Gauss elimination method for solving this difference scheme in the case of one-dimensional fractional parabolic partial differential equations.

\section{Stability of difference scheme}

Let us define the grid space

$$
\left\{\begin{array}{c}
\bar{\Omega}_{h}=\left\{x=x_{p}=\left(h_{1} p_{1}, \ldots, h_{m} p_{m}\right), p=\left(p_{1}, \ldots, p_{m}\right),\right. \\
\left.\quad 0 \leq p_{j} \leq M_{j}, h_{j} M_{j}=1, j=1, \ldots, m\right\}, \\
\Omega_{h}=\bar{\Omega}_{h} \cap \Omega, \quad S_{h}=\bar{\Omega}_{h} \cap S .
\end{array}\right.
$$

We introduce the Hilbert space $L_{2 h}=L_{2}\left(\bar{\Omega}_{h}\right)$ of the grid function $\varphi^{h}(x)=\left\{\varphi\left(h_{1} j_{1}, \ldots, h_{m} j_{m}\right)\right\}$ defined on $\bar{\Omega}$, equipped with the norm

$$
\left\|\varphi^{h}\right\|_{L_{2}\left(\bar{\Omega}_{h}\right)}=\left(\sum_{x \in \bar{\Omega}_{h}}\left|\varphi^{h}(x)\right|^{2} h_{1} \cdots h_{m}\right)^{1 / 2} .
$$

To the differential operator $A^{x}$ generated by problem (1.1) or (1.2), respectively, we assign the difference operator $A_{h}^{x}$ by the formula

$$
A_{h}^{x} u^{h}=-\sum_{p=1}^{m}\left(a_{p}(x) u_{\bar{x}_{p}}^{h}\right)_{x_{p}, j_{p}}+\sigma u^{h}
$$

acting in the space of grid functions $u^{h}(x)$, satisfying the conditions $u^{h}(x)=0$ or $\frac{\partial u(t, x)}{\partial \vec{n}}=0$ $\left(\forall x \in S_{h}\right)$. It is known that $A_{h}^{x}$ is a self-adjoint positive definite operator in $L_{2}\left(\bar{\Omega}_{h}\right)$. Here,

$$
\begin{aligned}
\varphi_{x_{p}, j_{p}} & =\frac{1}{h_{p}}\left(\varphi\left(h_{1} j_{1}, \ldots, h_{j}\left(j_{j}+1\right), \ldots, h_{m} j_{m}\right)-\varphi\left(h_{1} j_{1}, \ldots, h_{j} j_{j}, \ldots, h_{m} j_{m}\right)\right), \\
\varphi_{\bar{x}_{p}, j_{p}} & =\frac{1}{h_{p}}\left(\varphi\left(h_{1} j_{1}, \ldots, h_{j} j_{j}, \ldots, h_{m} j_{m}\right)-\varphi\left(h_{1} j_{1}, \ldots, h_{j}\left(j_{j}-1\right), \ldots, h_{m} j_{m}\right)\right) .
\end{aligned}
$$


With the help of $A_{h}^{x}$, we arrive at the initial boundary value problem

$$
\left\{\begin{array}{l}
\frac{d v^{h}(t, x)}{d t}+D_{t}^{1 / 2} v^{h}(t, x)+A_{h}^{x} v^{h}(t, x)=f^{h}(t, x), \quad 0<t<T, x \in \Omega_{h}, \\
v^{h}(0, x)=0, \quad x \in \bar{\Omega}
\end{array}\right.
$$

for a finite system of ordinary fractional differential equations.

We denote

$$
d=\frac{2}{\sqrt{\pi \tau}}, \quad \lambda(q)=\sqrt{q+1 / 2}-\sqrt{q-1 / 2}, \quad \mu(q)=-\frac{1}{3}\left((q+1 / 2)^{3 / 2}-(q-1 / 2)^{3 / 2}\right) .
$$

Applying the second order of the approximation formula

$$
D_{t_{k-\frac{\tau}{2}}}^{1 / 2} u_{k}=d \sum_{i=0}^{k} \alpha_{k, i} u_{i}
$$

for

$$
D_{t_{k-\frac{\tau}{2}}}^{1 / 2} u\left(t_{k}-\tau / 2\right)=\frac{1}{\Gamma(1 / 2)} \int_{0}^{t_{k}-\tau / 2}\left(t_{k}-\tau / 2-s\right)^{-1 / 2} u^{\prime}(s) d s
$$

(see [16]) and the Crank-Nicholson difference scheme for parabolic equations, one can present the second order of accuracy difference scheme with respect to $t$ and to $x$. Here,

$$
\begin{aligned}
\alpha_{1,0} & =-\sqrt{2} / 3, \quad \alpha_{1,1}=\sqrt{2} / 3, \quad k=1, \\
\alpha_{2,0}= & -2 \sqrt{6} / 5, \quad \alpha_{2,1}=\sqrt{6} / 5, \quad \alpha_{2,2}=\sqrt{6} / 5, \quad k=2, \\
\alpha_{k, 0}= & (k-2) \lambda(k-2)+\mu(k-2), \\
\alpha_{k, 1}= & (k-3) \lambda(k-3)+\mu(k-3)+(3-2 k) \lambda(k-2)-2 \mu(k-2), \\
\alpha_{k, i}= & (k-i-2) \lambda(k-i-2)+\mu(k-i-2) \\
& +(2 i-2 k+1) \lambda(k-i-1)-2 \mu(k-i-1) \\
& +(k-i+1) \lambda(k-i)+\mu(k-i), \quad 2 \leq i \leq k-3, \\
\alpha_{k, k-2} & =-3 \lambda(1)-2 \mu(1)+3 \lambda(2)+\mu(2)-\frac{1}{6 \sqrt{2}}, \\
\alpha_{k, k-1} & =2 \lambda(1)+\mu(1)-\frac{\sqrt{2}}{3}, \quad 3 \leq k \leq N .
\end{aligned}
$$

Now, we introduce the second order accuracy $r$-modified Crank-Nicholson difference scheme in the following form:

$$
\left\{\begin{array}{l}
\frac{u_{k}^{h}(x)-u_{k-1}^{h}(x)}{\tau}+D_{t_{k}}^{1 / 2} u_{k}^{h}(x)+A_{h}^{x} u_{k}^{h}(x)=f_{k}^{h}(x), \quad x \in \bar{\Omega}_{h}, 1 \leq k \leq r, \\
\frac{u_{k}^{h}(x)-u_{k-1}^{h}(x)}{\tau}+D_{t_{k}}^{1 / 2} u_{k}^{h}(x)+\frac{1}{2} A_{h}^{x}\left[u_{k}^{h}(x)+u_{k-1}^{h}(x)\right] \\
\quad=f_{k}^{h}(x), \quad x \in \bar{\Omega}_{h}, r+1 \leq k \leq N, \\
f_{k}^{h}(x)=f\left(t_{k}-\frac{\tau}{2}, x\right), \quad N \tau=T, t_{k}=k \tau, 1 \leq k \leq N, \\
u_{0}^{h}(x)=0, \quad x \in \bar{\Omega}_{h}
\end{array}\right.
$$

for the approximate solution of problem (2.2). 
Theorem 2.1 Let $\tau$ and $|h|$ be sufficiently small positive numbers. Then the solutions of the difference scheme (2.4) satisfy the following stability estimate:

$$
\max _{1 \leq k \leq N}\left\|u_{k}^{h}\right\|_{L_{2 h}} \leq C \max _{1 \leq k \leq N}\left\|f_{k}^{h}\right\|_{L_{2 h}}
$$

where $C$ does not depend on $\tau, h$, and $f_{k}^{h}, 1 \leq k \leq N$.

Proof Consider the difference scheme (2.4). We have

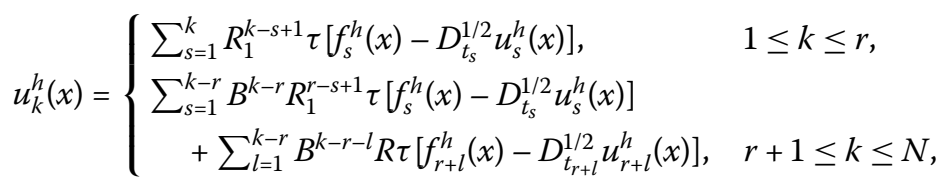

where

$$
\begin{aligned}
& R_{1}^{-1}=\left(I+\tau A_{h}^{x}\right), \\
& R^{-1}=\left(I+\frac{\tau}{2} A_{h}^{x}\right), \\
& B=R\left(I-\frac{\tau}{2} A_{h}^{x}\right) .
\end{aligned}
$$

We obtain

$$
\max _{1 \leq k \leq N}\left\|D_{t_{k}}^{1 / 2} u_{k}^{h}\right\|_{L_{2 h}} \leq M \max _{1 \leq k \leq N}\left\|f_{k}^{h}\right\|_{L_{2 h}}
$$

Let us write $z_{k}=\left\|D_{t_{k}}^{1 / 2} u_{k}^{h}\right\|_{L_{2 h}}$. Using (2.6), we have

$$
D_{t_{k}}^{1 / 2} u_{k}^{h}(x)=\left\{\begin{array}{ccc}
d \sum_{i=0}^{k} \alpha_{k, i}\left[\sum_{s=1}^{i} R_{1}^{i-s+1} \tau\left(f_{s}^{h}(x)-D_{t_{s}}^{1 / 2} u_{s}^{h}(x)\right)\right], & 1 \leq k \leq r, \\
d \sum_{i=0}^{k} \alpha_{k, i}\left[\sum_{s=1}^{i-r} B^{i-r} R_{1}^{r-s+1} \tau\left(f_{s}^{h}(x)-D_{t_{s}}^{1 / 2} u_{s}^{h}(x)\right)\right. & \\
\left.\quad+\sum_{l=1}^{i-r} B^{i-r-l} R \tau\left(f_{r+l}^{h}(x)-D_{t_{r+l}}^{1 / 2} u_{r+l}^{h}(x)\right)\right], & r+1 \leq k \leq N .
\end{array}\right.
$$

Now, let us estimate $z_{k}=\left\|D_{t_{k}}^{1 / 2} u_{k}^{h}\right\|_{L_{2 h}}, 1 \leq k \leq N$. From the triangle inequality, it follows that

$$
\begin{aligned}
z_{1} & \leq\left\|\alpha_{1,1} R_{1}\right\|_{L_{2 h} \rightarrow L_{2 h}}\left(\left\|f_{1}^{h}(x)\right\|_{L_{2 h}}+\left\|D_{\tau}^{1 / 2} u_{1}\right\|_{L_{2 h}}\right) \sqrt{\tau} \\
& \leq M_{1} \sqrt{\tau}\left(\left\|f_{1}^{h}(x)\right\|_{L_{2 h}}+z_{1}\right) .
\end{aligned}
$$

Applying the triangle inequality and the estimates [24]

$$
\begin{aligned}
& \left\|R_{1}^{k}\right\|_{L_{2 h} \rightarrow L_{2 h}} \leq \frac{M}{k \tau}, \\
& \left\|B^{i-r} R_{1}^{r-s+1}\right\|_{L_{2 h} \rightarrow L_{2 h}} \leq \frac{M}{k \tau}, \\
& \left\|B^{i-r-l} R\right\|_{L_{2 h} \rightarrow L_{2 h}} \leq M, \quad 1 \leq k \leq N,
\end{aligned}
$$


we have

$$
\begin{aligned}
z_{k} \leq & \sum_{i=0}^{k} \sum_{s=1}^{i}\left\|\alpha_{k, i} R_{1}^{i-s+1}\right\|_{L_{2 h} \rightarrow L_{2 h}}\left(\left\|f_{s}^{h}(x)\right\|_{L_{2 h}}+\left\|D_{t_{s}}^{1 / 2} u_{s}^{h}(x)\right\|_{L_{2 h}}\right) \sqrt{\tau} \\
\leq & M_{2} \sum_{s=1}^{k-1}\left[\frac{1}{\sqrt{(k-s) \tau}} \tau\left(\left\|f_{s}^{h}(x)\right\|_{L_{2 h}}+z_{s}\right)\right] \\
& +M_{3}\left(\left\|f_{k}^{h}(x)\right\|_{L_{2 h}}+z_{k}\right) \sqrt{\tau}, \quad 2 \leq k \leq r, \\
z_{k} \leq & \sum_{i=0}^{k} \sum_{s=1}^{i-r}\left\|\alpha_{k, i} B^{i-r} R_{1}^{r-s+1}\right\|_{L_{2 h} \rightarrow L_{2 h}}\left(\left\|f_{s}^{h}(x)\right\|_{L_{2 h}}+\left\|D_{t_{s}}^{1 / 2} u_{s}^{h}(x)\right\|_{L_{2 h}}\right) \sqrt{\tau} \\
& +\sum_{i=0}^{k} \sum_{l=1}^{i-r}\left\|\alpha_{k, i} B^{i-r-l} R\right\|_{L_{2 h} \rightarrow L_{2 h}}\left(\left\|f_{r+l}^{h}(x)\right\|_{L_{2 h}}+\left\|D_{t_{r+l}}^{1 / 2} u_{r+l}^{h}(x)\right\|_{L_{2 h}}\right) \sqrt{\tau} \\
\leq & M_{2}^{\prime} \sum_{s=1}^{k-1}\left[\frac{1}{\sqrt{(k-s) \tau}} \tau\left(\left\|f_{s}^{h}(x)\right\|_{L_{2 h}}+z_{s}\right)\right] \\
& +M_{3}^{\prime}\left(\left\|f_{k}^{h}(x)\right\|_{L_{2 h}}+z_{k}\right) \sqrt{\tau} \\
& +M_{2}^{\prime \prime} \sum_{l=1}^{k-1}\left[\frac{1}{\sqrt{(k-s) \tau}} \tau\left(\left\|f_{r+l}^{h}(x)\right\|_{L_{2 h}}+z_{r+l}\right)\right] \\
& +M_{3}^{\prime \prime}\left(\left\|f_{k}^{h}(x)\right\|_{L_{2 h}}+z_{k}\right) \sqrt{\tau}, \quad r+1 \leq k \leq N .
\end{aligned}
$$

Hence, applying the difference analog of the integral inequality and inequalities (2.8), (2.10), and (2.11), we get

$$
\begin{aligned}
\left\|\left\{z_{k}\right\}_{1}^{N}\right\|_{L_{2 h}} & =\left\|\left\{D_{\tau}^{\frac{1}{2}} u_{k}\right\}_{1}^{N}\right\|_{L_{2 h}} \\
& \leq M\left\|f^{\tau}\right\|_{L_{2 h}} .
\end{aligned}
$$

The proof of estimate (2.5) for the solution of (2.4) follows from (2.6), (2.9), and (2.12). Note that $M_{*}, M_{0}$ are independent from $\tau, h$, and $f_{k}^{h}, 1 \leq k \leq N$. Theorem 2.1 is proved.

\section{Numerical analysis}

We consider two examples for numerical results.

Example 3.1 We consider the following initial boundary value problem with Dirichlet condition for the one-dimensional fractional parabolic partial differential equation:

$$
\left\{\begin{aligned}
\frac{\partial u(t, x)}{\partial t}+ & D_{t}^{1 / 2} u(t, x)-\frac{\partial}{\partial x}\left((1+x) \frac{\partial u(t, x)}{\partial x}\right)=f(t, x) \\
f(t, x)= & {\left[\frac{3 \sqrt{t}}{2}+\frac{3 \sqrt{\pi} t}{4}+(1+x) \pi^{2} t^{3 / 2}\right] \sin \pi x } \\
& -\pi t^{3 / 2} \cos \pi x, \quad 0<t<1,0<x<1 \\
u(t, 0)= & u(t, 1)=0, \quad 0 \leq t \leq 1 \\
u(0, x)= & 0, \quad 0 \leq x \leq 1
\end{aligned}\right.
$$


It is clear that the exact solution of problem (3.1) is

$$
u(t, x)=t^{3 / 2} \sin \pi x
$$

Applying the $r$-modified Crank-Nicholson difference scheme (2.4), we get

$$
\left\{\begin{array}{l}
\frac{u_{n}^{k}-u_{n}^{k-1}}{\tau}+D_{t_{k}-\frac{\tau}{2}}^{1 / 2} u_{n}^{k}-\left[\left(1+x_{n}\right) \frac{u_{n+1}^{k}-2 u_{n}^{k}+u_{n-1}^{k}}{h^{2}}+\frac{u_{n+1}^{k}-u_{n-1}^{k}}{2 h}\right]=\varphi_{n}^{k}, \quad 1 \leq k \leq r, \\
\frac{u_{n}^{k}-u_{n}^{k-1}}{\tau}+D_{t_{k}-\frac{\tau}{2}}^{1 / 2} u_{n}^{k}-\frac{1}{2}\left[\left(1+x_{n}\right) \frac{u_{n+1}^{k}-2 u_{n}^{k}+u_{n-1}^{k}}{h^{2}}+\frac{u_{n+1}^{k}-u_{n-1}^{k}}{2 h}\right. \\
\left.\quad+\left(1+x_{n}\right) \frac{u_{n+1}^{k-1}-2 u_{n}^{k-1}+u_{n-1}^{k-1}}{h^{2}}+\frac{u_{n+1}^{k-1}-u_{n-1}^{k-1}}{2 h}\right]=\varphi_{n}^{k}, \quad r+1 \leq k \leq N, \\
\varphi_{n}^{k}=f\left(t_{k}-\frac{\tau}{2}, x_{n}\right), \quad t_{k}=k \tau, x_{n}=n h, 1 \leq k \leq N, 1 \leq n \leq M-1, \\
u_{0}^{k}=u_{M}^{k}=0, \quad 0 \leq k \leq N, \\
u_{n}^{0}=0, \quad 0 \leq n \leq M,
\end{array}\right.
$$

where $D_{t_{k}-\frac{\tau}{2}}^{1 / 2} u_{n}^{k}$ is defined by (2.3) for any $n, 1 \leq n \leq M-1$. We can rewrite it in the system of equations with matrix coefficients

$$
\left\{\begin{array}{l}
A U_{n+1}+B U_{n}+C U_{n-1}=D \varphi_{n}, \quad 1 \leq n \leq M-1 \\
U_{0}=\tilde{0} \\
U_{M}=\tilde{0}
\end{array}\right.
$$

Here and in the sequel $\tilde{0}$ is the $(N+1) \times 1$ zero matrix,

$$
\begin{aligned}
& A=\left[\begin{array}{cccccccccccc}
0 & 0 & 0 & \cdots & 0 & 0 & 0 & 0 & \cdots & 0 & 0 & 0 \\
0 & a_{n} & 0 & \cdots & 0 & 0 & 0 & 0 & \cdots & 0 & 0 & 0 \\
0 & 0 & a_{n} & \cdots & 0 & 0 & 0 & 0 & \cdots & 0 & 0 & 0 \\
\vdots & \vdots & \vdots & \ddots & \vdots & \vdots & \vdots & \vdots & \ddots & \vdots & \vdots & \vdots \\
0 & 0 & 0 & \cdots & a_{n} & 0 & 0 & 0 & \cdots & 0 & 0 & 0 \\
0 & 0 & 0 & \cdots & 0 & a_{n} & 0 & 0 & \cdots & 0 & 0 & 0 \\
0 & 0 & 0 & \cdots & 0 & z_{n} & z_{n} & 0 & \cdots & 0 & 0 & 0 \\
0 & 0 & 0 & \cdots & 0 & 0 & z_{n} & z_{n} & \cdots & 0 & 0 & 0 \\
\vdots & \vdots & \vdots & \ddots & \vdots & \vdots & \vdots & \vdots & \ddots & \vdots & \vdots & \vdots \\
0 & 0 & 0 & \cdots & 0 & 0 & 0 & 0 & \cdots & z_{n} & z_{n} & 0 \\
0 & 0 & 0 & \cdots & 0 & 0 & 0 & 0 & \cdots & 0 & z_{n} & z_{n}
\end{array}\right]_{(N+1) \times(N+1)} \\
& B=B_{1}+B_{2} \text {, } \\
& B_{1}=\left[\begin{array}{cccccc}
b_{11} & 0 & 0 & \cdots & 0 & 0 \\
b_{21} & b_{22} & 0 & \cdots & 0 & 0 \\
b_{31} & b_{32} & b_{33} & \cdots & 0 & 0 \\
\vdots & \vdots & \vdots & \ddots & \vdots & \vdots \\
b_{N, 1} & b_{N, 2} & b_{N, 3} & \cdots & b_{N, N} & 0 \\
b_{N+1,1} & b_{N+1,2} & b_{N+1,3} & \cdots & b_{N+1, N} & b_{N+1, N+1}
\end{array}\right]_{(N+1) \times(N+1)}
\end{aligned}
$$




$$
\begin{aligned}
& B_{2}=\left[\begin{array}{cccccccccccc}
1 & 0 & 0 & \cdots & 0 & 0 & 0 & 0 & \cdots & 0 & 0 & 0 \\
-1 / \tau & \eta_{n} & 0 & \cdots & 0 & 0 & 0 & 0 & \cdots & 0 & 0 & 0 \\
0 & -1 / \tau & \eta_{n} & \cdots & 0 & 0 & 0 & 0 & \cdots & 0 & 0 & 0 \\
\vdots & \vdots & \vdots & \ddots & \vdots & \vdots & \vdots & \vdots & \ddots & \vdots & \vdots & \vdots \\
0 & 0 & 0 & \cdots & \eta_{n} & 0 & 0 & 0 & \cdots & 0 & 0 & 0 \\
0 & 0 & 0 & \cdots & -1 / \tau & \eta_{n} & 0 & 0 & \cdots & 0 & 0 & 0 \\
0 & 0 & 0 & \cdots & 0 & v_{n} & w_{n} & 0 & \cdots & 0 & 0 & 0 \\
0 & 0 & 0 & \cdots & 0 & 0 & v_{n} & w_{n} & \cdots & 0 & 0 & 0 \\
\vdots & \vdots & \vdots & \ddots & \vdots & \vdots & \vdots & \vdots & \ddots & \vdots & \vdots & \vdots \\
0 & 0 & 0 & \cdots & 0 & 0 & 0 & 0 & \cdots & v_{n} & w_{n} & 0 \\
0 & 0 & 0 & \cdots & 0 & 0 & 0 & 0 & \cdots & 0 & v_{n} & w_{n}
\end{array}\right]_{(N+1) \times(N+1)} \\
& C=\left[\begin{array}{cccccccccccc}
0 & 0 & 0 & \cdots & 0 & 0 & 0 & 0 & \cdots & 0 & 0 & 0 \\
0 & c_{n} & 0 & \cdots & 0 & 0 & 0 & 0 & \cdots & 0 & 0 & 0 \\
0 & 0 & c_{n} & \cdots & 0 & 0 & 0 & 0 & \cdots & 0 & 0 & 0 \\
\vdots & \vdots & \vdots & \ddots & \vdots & \vdots & \vdots & \vdots & \ddots & \vdots & \vdots & \vdots \\
0 & 0 & 0 & \cdots & c_{n} & 0 & 0 & 0 & \cdots & 0 & 0 & 0 \\
0 & 0 & 0 & \cdots & 0 & c_{n} & 0 & 0 & \cdots & 0 & 0 & 0 \\
0 & 0 & 0 & \cdots & 0 & y_{n} & y_{n} & 0 & \cdots & 0 & 0 & 0 \\
0 & 0 & 0 & \cdots & 0 & 0 & y_{n} & y_{n} & \cdots & 0 & 0 & 0 \\
\vdots & \vdots & \vdots & \ddots & \vdots & \vdots & \vdots & \vdots & \ddots & \vdots & \vdots & \vdots \\
0 & 0 & 0 & \cdots & 0 & 0 & 0 & 0 & \cdots & y_{n} & y_{n} & 0 \\
0 & 0 & 0 & \cdots & 0 & 0 & 0 & 0 & \cdots & 0 & y_{n} & y_{n}
\end{array}\right]_{(N+1) \times(N+1)} \\
& \varphi_{n}=\left[\begin{array}{c}
\varphi_{n}^{0} \\
\varphi_{n}^{1} \\
\varphi_{n}^{2} \\
\vdots \\
\varphi_{n}^{N-1} \\
\varphi_{n}^{N}
\end{array}\right]_{(N+1) \times 1}, \quad U_{q}=\left[\begin{array}{c}
u_{q}^{0} \\
u_{q}^{1} \\
u_{q}^{2} \\
\vdots \\
u_{q}^{N-1} \\
u_{q}^{N}
\end{array}\right]_{(N+1) \times 1} \quad, \quad q=n \pm 1, n, \\
& a_{n}=-\frac{1+x_{n}}{h^{2}}-\frac{1}{2 h}, \quad c_{n}=-\frac{1+x_{n}}{h^{2}}+\frac{1}{2 h}, \\
& z_{n}=-\frac{1}{2}\left(\frac{1+x_{n}}{h^{2}}+\frac{1}{2 h}\right), \quad y_{n}=-\frac{1}{2}\left(\frac{1+x_{n}}{h^{2}}-\frac{1}{2 h}\right) \text {, } \\
& \eta_{n}=\frac{1}{\tau}+\frac{2\left(1+x_{n}\right)}{h^{2}} \\
& v_{n}=-\frac{1}{\tau}+\frac{1+x_{n}}{h^{2}}, \quad w_{n}=\frac{1}{\tau}+\frac{1+x_{n}}{h^{2}}, \\
& b_{11}=1, \quad b_{21}=-\frac{2 \sqrt{2}}{3 \sqrt{\pi \tau}}, \quad b_{22}=\frac{2 \sqrt{2}}{3 \sqrt{\pi \tau}}, \\
& b_{31}=-\frac{4 \sqrt{6}}{5 \sqrt{\pi \tau}}, \quad b_{32}=\frac{2 \sqrt{6}}{5 \sqrt{\pi \tau}}, \quad b_{33}=\frac{2 \sqrt{6}}{5 \sqrt{\pi \tau}}, \\
& b_{41}=d[1 \lambda(1)+\mu(1)], \quad b_{42}=d[-3 \lambda(1)-2 \mu(1)]-d / 6 \sqrt{2} \text {, }
\end{aligned}
$$




$$
\begin{aligned}
& b_{43}=d[2 \lambda(1)+\mu(1)]-4 d / 6 \sqrt{2}, \quad b_{44}=5 d / 6 \sqrt{2}, \\
& b_{51}=d[2 \lambda(2)+\mu(2)], \quad b_{52}=d[-5 \lambda(2)-2 \mu(2)+1 \lambda(1)+\mu(1)], \\
& b_{53}=d[3 \lambda(2)+\mu(2)-3 \lambda(1)-2 \mu(1)]-d / 6 \sqrt{2}, \\
& b_{54}=d[2 \lambda(1)+\mu(1)]-4 d / 6 \sqrt{2}, \quad b_{55}=5 d / 6 \sqrt{2}, \\
& b_{i j}= \begin{cases}d[(i-3) \lambda(i-3)+\mu(i-3)], & j=1, \\
d[(5-2 i) \lambda(i-3)-2 \mu(i-3)+(i-4) \lambda(i-4)+\mu(i-4)], & j=2, \\
d[(i-j+1) \lambda(i-j)+\mu(i-j)+(2 j-2 i+1) \lambda(i-j-1) & 3 \leq 3 \leq 1 \leq i-3, \\
d[3 \lambda(2)+\mu(2)-3 \lambda(1)-2 \mu(1)]-d / 6 \sqrt{2}, & j=i-2, \\
5 d / 6 \sqrt{2}, & j=i-1, \\
0, & j=i,\end{cases}
\end{aligned}
$$

for $i=6,7, \ldots, N+1$, and

$$
\varphi_{n}^{k}=\left[\frac{3 \sqrt{k \tau}}{2}+\frac{3 \sqrt{\pi} k \tau}{4}+\pi^{2}(k \tau)^{3 / 2}(1+n h)\right] \sin (\pi n h)-\pi(k \tau)^{3 / 2} \cos (\pi n h) .
$$

For solving (3.2) we use a modified Gauss elimination method [27]. Hence, we seek a solution of the matrix equation in the following form:

$$
U_{j}=\alpha_{j+1} U_{j+1}+\beta_{j+1}, \quad j=M-1, \ldots, 2,1,
$$

where $\alpha_{j}(j=1,2, \ldots, M)$ are $(N+1) \times(N+1)$ square matrices and $\beta_{j}(j=1,2, \ldots, M)$ are $(N+1) \times 1$ column matrices defined by

$$
\begin{aligned}
& \alpha_{j+1}=-\left(B+C \alpha_{j}\right)^{-1} A, \\
& \beta_{j+1}=\left(B+C \alpha_{j}\right)^{-1}\left(D \varphi_{j}-C \beta_{j}\right), \quad j=1,2, \ldots, M-1,
\end{aligned}
$$

where $j=1,2, \ldots, M-1, \alpha_{1}$ is the $(N+1) \times(N+1)$ zero matrix and $\beta_{1}$ is the $(N+1) \times 1$ zero matrix and $U_{M}=0$.

Example 3.2 We consider the following initial boundary value problem with Neumann condition for the one-dimensional fractional parabolic partial differential equation:

$$
\left\{\begin{array}{l}
\frac{\partial u(t, x)}{\partial t}+D_{t}^{1 / 2} u(t, x)-\frac{\partial}{\partial x}\left((1+x) \frac{\partial u(t, x)}{\partial x}\right)+u(t, x)=f(t, x), \\
f(t, x)=\left(3+t+\frac{16 \sqrt{t}}{5 \sqrt{\pi}}+\pi^{2} t(1+x)\right) t^{2} \cos \pi x+\pi t^{3} \sin \pi x, \quad 0<t<1,0<x<1, \\
u_{x}(t, 0)=u_{x}(t, 1)=0, \quad 0 \leq t \leq 1, \\
u(0, x)=0, \quad 0 \leq x \leq 1 .
\end{array}\right.
$$

It is clear that the exact solution of problem (3.10) is

$$
u(t, x)=t^{3} \cos \pi x
$$


Applying the $r$-modified Crank-Nicholson difference scheme (2.4), we get

$$
\left\{\begin{array}{l}
\frac{u_{n}^{k}-u_{n}^{k-1}}{\tau}+D_{t_{k}-\frac{\tau}{2}}^{1 / 2} u_{n}^{k}-\left[\left(1+x_{n}\right) \frac{u_{n+1}^{k}-2 u_{n}^{k}+u_{n-1}^{k}}{h^{2}}+\frac{u_{n+1}^{k}-u_{n-1}^{k}}{2 h}+u_{n}^{k}\right]=\varphi_{n}^{k}, \quad 1 \leq k \leq r, \\
\frac{u_{n}^{k}-u_{n}^{k-1}}{\tau}+D_{t_{k}-\frac{\tau}{2}}^{1 / 2} u_{n}^{k}-\frac{1}{2}\left[\left(1+x_{n}\right) \frac{u_{n+1}^{k}-2 u_{n}^{k}+u_{n-1}^{k}}{h^{2}}+\frac{u_{n+1}^{k}-u_{n-1}^{k}}{2 h}+u_{n}^{k}\right. \\
\left.\quad+\left(1+x_{n}\right) \frac{u_{n+1}^{k-1}-2 u_{n}^{k-1}+u_{n-1}^{k-1}}{h^{2}}+\frac{u_{n+1}^{k-1}-u_{n-1}^{k-1}}{2 h}+u_{n}^{k-1}\right]=\varphi_{n}^{k}, \quad r+1 \leq k \leq N, \\
\varphi_{n}^{k}=f\left(t_{k}-\frac{\tau}{2}, x_{n}\right), \quad t_{k}=k \tau, x_{n}=n h, 1 \leq k \leq N, 1 \leq n \leq M-1, \\
u_{0}^{0}=0, \quad k=0, \\
-\frac{h}{4 \tau} u_{0}^{k-1}+\left[\frac{1}{h}+\frac{h}{2} D_{t}^{1 / 2}+\frac{h}{2}\right] u_{0}^{k}+\frac{h}{4 \tau} u_{0}^{k+1}=\frac{1}{h} u_{1}^{k}+\frac{h}{2} \varphi_{0}^{k}, \quad 1 \leq k \leq N-1, \\
\frac{h}{4 \tau} u_{0}^{N-2}+\frac{h}{\tau} u_{0}^{N-1}+\left[\frac{1}{h}+\frac{3 h}{4 \tau}+\frac{h}{2} D_{t}^{1 / 2}+\frac{h}{2}\right] u_{0}^{N}=\frac{1}{h} u_{1}^{N}+\frac{h}{2} \varphi_{0}^{N}, \quad k=N, \\
3 u_{M}^{k}-4 u_{M-1}^{k}+u_{M-2}^{k}=0, \quad 0 \leq k \leq N, \\
u_{n}^{0}=0, \quad 0 \leq n \leq M,
\end{array}\right.
$$

where $D_{t_{k}-\frac{\tau}{2}}^{1 / 2} u_{n}^{k}$ is defined by (2.3). We can rewrite it in the form of a system of equations with matrix coefficients

$$
\left\{\begin{array}{l}
A U_{n+1}+B U_{n}+C U_{n-1}=D \varphi_{n}, \quad 1 \leq n \leq M-1, \\
E U_{0}=F U_{1}+R \varphi_{0}, \quad 3 U_{M}-4 U_{M-1}+U_{M-2}=\tilde{0}
\end{array}\right.
$$

Here, $A, B, C$ are defined by (3.3), (3.4), and (3.5):

$$
\begin{aligned}
& F=\left[\begin{array}{cccccc}
0 & 0 & 0 & \cdots & 0 & 0 \\
0 & 1 / h & 0 & \cdots & 0 & 0 \\
0 & 0 & 1 / h & \cdots & 0 & 0 \\
\cdots & \cdots & \cdots & \cdots & \cdots & \cdots \\
0 & 0 & 0 & \cdots & 1 / h & 0 \\
0 & 0 & 0 & \cdots & 0 & 1 / h
\end{array}\right]_{(N+1) \times(N+1)} \\
& R=\left[\begin{array}{cccccc}
0 & 0 & 0 & \cdots & 0 & 0 \\
0 & h / 2 & 0 & \cdots & 0 & 0 \\
0 & 0 & h / 2 & \cdots & 0 & 0 \\
\cdots & \cdots & \cdots & \cdots & \cdots & \cdots \\
0 & 0 & 0 & \cdots & h / 2 & 0 \\
0 & 0 & 0 & \cdots & 0 & h / 2
\end{array}\right]_{(N+1) \times(N+1)} \\
& E=\left[\begin{array}{cccccc}
e_{11} & 0 & 0 & \cdots & 0 & 0 \\
e_{21} & e_{22} & 0 & \cdots & 0 & 0 \\
e_{31} & e_{32} & e_{33} & \cdots & 0 & 0 \\
\cdots & \cdots & \cdots & \cdots & \cdots & \cdots \\
e_{N 1} & e_{N 2} & e_{N 3} & \cdots & e_{N N} & 0 \\
e_{N+1,1} & e_{N+1,2} & e_{N+1,3} & \cdots & e_{N+1, N} & e_{N+1, N+1}
\end{array}\right]_{(N+1) \times(N+1)} \\
& e_{11}=1, \quad e_{21}=-\frac{h}{4 \tau}-\frac{4 h}{3 \sqrt{\pi \tau}}, \quad e_{22}=\frac{1}{h}+\frac{h}{2}+\frac{4 h}{3 \sqrt{\pi \tau}}, \quad e_{23}=\frac{h}{4 \tau}, \\
& e_{31}=\frac{2 \sqrt{2} h}{15 \sqrt{\pi \tau}}, \quad e_{32}=\frac{-16 \sqrt{2} h}{15 \sqrt{\pi \tau}}-\frac{h}{4 \tau}, \quad e_{33}=\frac{1}{h}+\frac{h}{2}+\frac{14 \sqrt{2} h}{15 \sqrt{\pi \tau}}, \quad e_{34}=\frac{h}{4 \tau}, \\
& e_{41}=\frac{d h}{2}[(1+1 / 2) \lambda(1)+\mu(1)], \quad e_{42}=\frac{d h}{2}[-4 \lambda(1)-2 \mu(1)+1 / 2 \lambda(0)+\mu(0)],
\end{aligned}
$$




$$
\begin{aligned}
& e_{43}=-\frac{h}{4 \tau}+\frac{d h}{2}[(2+1 / 2) \lambda(1)+\mu(1)-2-2(-1 / 3)] \text {, } \\
& e_{44}=\frac{1}{h}+\frac{h}{2}+\frac{d h}{2}[(1+1 / 2) \lambda(0)+\mu(0)], \quad e_{45}=\frac{h}{4 \tau}, \\
& e_{51}=\frac{d h}{2}[(2+1 / 2) \lambda(2)+\mu(2)] \text {, } \\
& e_{52}=\frac{d h}{2}[-2 \cdot 3 \lambda(2)-2 \mu(2)+(1+1 / 2) \lambda(1)+\mu(1)] \text {, } \\
& e_{53}=\frac{d h}{2}[(2+1+1 / 2) \lambda(2)+\mu(2)-2 \cdot 2 \lambda(1)-2 \mu(1)+1 / 2 \lambda(0)+\mu(0)] \text {, } \\
& e_{54}=-\frac{h}{4 \tau}+\frac{d h}{2}[(1+1+1 / 2) \lambda(1)+\mu(1)-2 \lambda(0)-2 \mu(0)] \\
& e_{55}=\frac{1}{h}+\frac{h}{2}+\frac{d h}{2}[(1+1 / 2) \lambda(0)+\mu(0)], \quad e_{56}=\frac{h}{4 \tau}, \\
& \left\{\begin{array}{lr}
\frac{d h}{2}[(i-3+1 / 2) \lambda(i-3)+\mu(i-3)], & j=1, \\
\frac{d h}{2}[-2(i-2) \lambda(i-3)-2 \mu(i-3)+(i-4+1 / 2) \lambda(i-4)+\mu(i-4)], & j=2
\end{array}\right. \\
& \frac{d h}{2}[(i-j+1+1 / 2) \lambda(i-j)+\mu(i-j)-2(i-j) \lambda(i-j-1) \\
& -2 \mu(i-j-1)+(i-j-2+1 / 2) \lambda(i-j-2)+\mu(i-j-2)], \quad 3 \leq j \leq i-2, \\
& e_{i j}= \begin{cases}\frac{h}{4 \tau}+\frac{d h}{2}[(2+1 / 2) \lambda(1)+\mu(1)-2 \lambda(0)-2 \mu(0)], & j=i-1, \\
\frac{1}{h}+\frac{h}{2}+\frac{d h}{2}[(1+1 / 2) \lambda(0)+\mu(0)], & j=i, \\
\frac{h}{4 \tau}, & j=i+1,\end{cases} \\
& \frac{h}{4 \tau}+\frac{d h}{2}[(i-N+2+1 / 2) \lambda(i-N+1)+\mu(i-N+1) \\
& -2(i-N+1) \lambda(i-N)-2 \mu(i-N) \\
& +(i-N-1+1 / 2) \lambda(i-N-1)+\mu(i-N-1)], \quad j=N-1, \\
& -\frac{h}{\tau}+\frac{d h}{2}[(2+1 / 2) \lambda(1)+\mu(1)-2 \lambda(0)-2 \mu(0)], \quad j=N, \\
& \begin{array}{ll}
\frac{1}{h}+\frac{h}{2}+\frac{3 h}{4 \tau}+\frac{d h}{2}[(1+1 / 2) \lambda(0)+\mu(0)], & j=N+1 \\
0, & j>i+1
\end{array} \\
& 0 \text {, }
\end{aligned}
$$

for $i=6,7, \ldots, N+1$, and

$$
\begin{aligned}
\varphi_{0}^{k} & =\left(3+k \tau+\frac{16 \sqrt{k \tau}}{5 \sqrt{\pi}}+\pi^{2} k \tau\right)(k \tau)^{2}, \\
\varphi_{n}^{k} & =\left[3+k \tau+\frac{16 \sqrt{k \tau}}{5 \sqrt{\pi}}+\pi^{2}(k \tau)(1+n h)\right](k \tau)^{2} \cos (\pi n h)+\pi(k \tau)^{3} \sin (\pi n h) .
\end{aligned}
$$

For solving this matrix equation we will use the same method as for Example 3.1. Namely, we use (3.7), (3.8), (3.9), and

$$
\begin{aligned}
& u_{M}=\left[3 I-4 \alpha_{M}+\alpha_{M-1} \alpha_{M}\right]^{-1} *\left[\left(4 I-\alpha_{M-1}\right) \beta_{M}-\beta_{M-1}\right] \\
& \alpha_{1}=E^{-1} F, \quad \beta_{1}=E^{-1} R \varphi_{0} .
\end{aligned}
$$

Finally, we give the results of the numerical analysis. The numerical solutions are recorded for different values of the modification parameter $r$, and discretization parameters $N$ and $M$. Besides $u_{n}^{k}$ represents the numerical solutions of these difference schemes at $\left(t_{k}, x_{n}\right)$. The error is computed by the following formula:

$$
E_{M}^{N}=\max _{1 \leq k \leq N, 1 \leq n \leq M-1}\left|u\left(t_{k}, x_{n}\right)-u_{n}^{k}\right| .
$$


Table 1 Error analysis for Dirichlet problem

\begin{tabular}{lcc}
\hline Method & $\mathbf{N =} \boldsymbol{M = 4 0}$ & $\boldsymbol{N}=\boldsymbol{M = 8 0}$ \\
\hline Rothe & 0.019436847 & 0.009569477 \\
Crank-Nicholson & 0.000525352 & 0.000149122 \\
One-modified Crank-Nicholson & 0.000525046 & 0.000149050 \\
Two-modified Crank-Nicholson & 0.000503791 & 0.000144560 \\
Three-modified Crank-Nicholson & 0.001309365 & 0.000260588 \\
\hline
\end{tabular}

Table 2 Error analysis for Neumann problem

\begin{tabular}{lll}
\hline Method & $\mathbf{N =} \boldsymbol{M = 4 0}$ & $\boldsymbol{N}=\boldsymbol{M = 8 0}$ \\
\hline Rothe & 0.038312769 & 0.018981758 \\
Crank-Nicholson & 0.009051376 & 0.002281280 \\
One-modified Crank-Nicholson & 0.011770581 & 0.002979532 \\
Two-modified Crank-Nicholson & 0.011770790 & 0.002979547 \\
Three-modified Crank-Nicholson & 0.011770831 & 0.002979551 \\
\hline
\end{tabular}

Table 1 and Table 2 are constructed for $N=M=40$ and 80, respectively. As can be seen from Table 1, the $r$-modified Crank-Nicholson difference scheme is more accurate than the Crank-Nicholson and Rothe difference schemes. Table 2 shows that the $r$-modified CrankNicholson difference scheme has the same order error as the Crank-Nicholson difference scheme.

\section{Conclusion}

In this study, the second order of accuracy stable difference scheme for the numerical solution of the mixed problem for the fractional parabolic equation is investigated. We have obtained a stability estimate for the solution of this difference scheme. The theoretical statements for the solution of this difference scheme for one-dimensional parabolic equations are supported by numerical examples obtained by computer.

\section{Competing interests}

The authors declare that they have no competing interests

\section{Authors' contributions}

ZC proposed the main idea of this paper, obtained the theoretical and numerical results. AA designed the study and interpreted the results. All authors read and approved the final manuscript.

\section{Author details}

${ }^{1}$ Department of Mathematics, Fatih University, Istanbul, Turkey. ${ }^{2}$ Department of Mathematics, ITTU, Ashgabat,

Turkmenistan. ${ }^{3}$ Department of Mathematical Engineering, Gumushane University, Gumushane, Turkey.

\section{Received: 2 December 2013 Accepted: 18 March 2014 Published: 31 Mar 2014}

\section{References}

1. Podlubny, I: Fractional Differential Equations. Mathematics in Science and Engineering, vol. 198. Academic Press, San Diego (1999)

2. Kilbas, AA, Srivastava, HM, Trujillo, JJ: Theory and Applications of Fractional Differential Equations, vol. 204. Elsevier, Amsterdam (2006)

3. Diethelm, K: The Analysis of Fractional Differential Equations. Springer, Berlin (2010)

4. Diethelm, K, Ford, NJ: Multi-order fractional differential equations and their numerical solution. Appl. Math. Comput. 154(3), 621-640 (2004). doi:10.1016/S0096-3003(03)00739-2

5. El-Sayed, AMA, El-Mesiry, AEM, El-Saka, HAA: Numerical solution for multi-term fractional (arbitrary) orders differential equations. Comput. Appl. Math. 23(1), 33-54 (2004)

6. De la Sen, M: Positivity and stability of the solutions of Caputo fractional linear time-invariant systems of any order with internal point delays. Abstr. Appl. Anal. 2011, Article ID 161246 (2011). doi:10.1155/2011/161246

7. Yakar, A, Koksal, ME: Existence results for solutions of nonlinear fractional differential equations. Abstr. Appl. Anal. 2012, Article ID 267108 (2012). doi:10.1155/2012/267108 
8. Yuan, C: Two positive solutions for $(n-1,1)$-type semipositone integral boundary value problems for coupled systems of nonlinear fractional differential equations. Commun. Nonlinear Sci. Numer. Simul. 17(2), 930-942 (2012). doi:10.1016/j.cnsns.2011.06.008

9. De la Sen, M, Agarwal, RP, Ibeas, A, Alonso-Quesada, S: On the existence of equilibrium points, boundedness, oscillating behavior and positivity of a SVEIRS epidemic model under constant and impulsive vaccination. Adv. Differ. Equ. 2011, Article ID 748608 (2011). doi:10.1155/2011/748608

10. Yuan, C: Multiple positive solutions for semipositone $(n, p)$-type boundary value problems of nonlinear fractional differential equations. Anal. Appl. 9(1), 97-112 (2011). doi:10.1142/S0219530511001753

11. Agarwal, RP, de Andrade, B, Cuevas, C: Weighted pseudo-almost periodic solutions of a class of semilinear fractional differential equations. Nonlinear Anal., Real World Appl. 11, 3532-3554 (2010). doi:10.1016/j.nonrwa.2010.01.002

12. Ashyralyev, A: A note on fractional derivatives and fractional powers of operators. J. Math. Anal. Appl. 357(1), 232-236 (2009). doi:10.1016/j.jmaa.2009.04.012

13. Ashyralyev, A, Dal, F, Pinar, Z: A note on fractional hyperbolic differential and difference equations. Appl. Math. Comput. 217(9), 4654-4664 (2011). doi:10.1016/j.amc.2010.11.017

14. Berdyshev, AS, Cabada, A, Karimov, ET: On a non-local boundary problem for a parabolic-hyperbolic equation involving a Riemann-Liouville fractional differential operator. Nonlinear Anal. 75(6), 3268-3273 (2011). doi:10.1016/j.na.2011.12.033

15. Amanov, D, Ashyralyev, A: Initial-boundary value problem for fractional partial differential equations of higher order. Abstr. Appl. Anal. 2012, Article ID 973102 (2012). doi:10.1155/2012/973102

16. Cakir, Z: Stability of difference schemes for fractional parabolic PDE with the Dirichlet-Neumann conditions. Abstr. Appl. Anal. 2012, Article ID 463746 (2012). doi:10.1155/2012/463746

17. Ashyralyev, A, Cakir, Z: On the numerical solution of fractional parabolic partial differential equations with the Dirichlet condition. Discrete Dyn. Nat. Soc. 2012, Article ID 696179 (2012). doi:10.1155/2012/696179

18. Ashyralyev, A, Cakir, Z: FDM for fractional parabolic equations with the Neumann condition. Adv. Differ. Equ. 2013, 120 (2013). doi:10.1186/1687-1847-2013-120

19. Ashyralyev, A, Hicdurmaz, B: A note on the fractional Schrodinger differential equations. Kybernetes 40(5-6), 736-750 (2011). doi:10.1108/03684921111142287

20. Ashyralyev, A: Well-posedness of the Basset problem in spaces of smooth functions. Appl. Math. Lett. 24(7), 1176-1180 (2011). doi:10.1016/j.aml.2011.02.002

21. Clement, P, Guerre-Delabrire, S: On the regularity of abstract Cauchy problems and boundary value problems. Atti Accad. Naz. Lincei, Rend. Lincei, Mat. Appl. 9(4), 245-266 (1999)

22. Agarwal, RP, Bohner, M, Shakhmurov, VB: Maximal regular boundary value problems in Banach-valued weighted spaces. Bound. Value Probl. 1, 9-42 (2005). doi:10.1155/BVP.2005.9

23. Lunardi, A: Analytic Semigroups and Optimal Regularity in Parabolic Problems. Operator Theory: Advances and Applications. Birkhäuser, Basel (1995)

24. Ashyralyev, A, Sobolevskii, PE: Well-Posedness of Parabolic Difference Equations. Birkhäuser, Basel (1994)

25. Rassias, JM, Karimov, ET: Boundary-value problems with non-local condition for degenerate parabolic equations. Contemp. Anal. Appl. Math. 1(1), 42-48 (2013)

26. Selitskii, AM: The space of initial data for the second boundary-value problem for parabolic differential-difference equation. Contemp. Anal. Appl. Math. 1(1), 34-41 (2013)

27. Samarskii, AA, Nikolaev, ES: Numerical Methods for Grid Equations. Iterative Methods, vol. 2. Birkhäuser, Basel (1989)

10.1186/1687-2770-2014-76

Cite this article as: Ashyralyev and Cakir: r-Modified Crank-Nicholson difference scheme for fractional parabolic PDE. Boundary Value Problems 2014, 2014:76

\section{Submit your manuscript to a SpringerOpen ${ }^{\circ}$ journal and benefit from:}

- Convenient online submission

- Rigorous peer review

Immediate publication on acceptance

- Open access: articles freely available online

- High visibility within the field

- Retaining the copyright to your article 